Vorreiter in Energie, Mobilität und Abfall

\title{
Transformationspioniere für eine klimafreundliche Stadt
}

\author{
In sozial-ökologischen Transformationsprozessen \\ nehmen Pioniere des Wandels eine Schlüssel- \\ rolle ein. Auf Basis qualitativer Interviews wird \\ analysiert, welche förderlichen und hinderlichen \\ Bedingungen auf das Wirken und die Ver- \\ breitung von Pionieraktivitäten Einfluss nehmen. \\ Von Toya Engel, Katharina Klindworth und \\ Jörg Knieling
}

\section{Einführung}

Eine umfassende Transformation der Gesellschaft erscheint vor dem Hintergrund der veränderten und absehbaren Umweltbedingungen unausweichlich (Welzer et al. 2014; WBGU 2011).Für die notwendigen gesellschaftlichen Veränderungen in Transformationsprozessen wird Pionieren des Wandels eine Schlüsselrolle zugeschrieben (WBGU 2011; Kristof 2010). Sie können mit ihren Aktivitäten als Individuen, Gruppen, Vereine, NGOs oder Unternehmen besondere Wirkung in Richtung der für eine gesamtgesellschaftliche Transformation zur Nachhaltigkeit erforderlichen Innovationen entfalten (Gibbs et al. 2014; WBGU 2011; 2016).

Im Rahmen einer qualitativen Fallstudienanalyse zu Transformationspionieren wurden in den drei Handlungsfeldern des Klimaschutzes Energie, Mobilität und Abfall in Hamburg mittels Desktoprecherche und Experteninterviews mehr als 120 Transformationspioniere identifiziert und 20 leitfadengestützte Interviews qualitativ interpretiert (Fenzl/Mayring 2014). Das Erkenntnisinteresse der Analyse lag auf den Motiven für die Aktivitäten der Pioniere, ihren Zielen sowie den förderlichen und hemmenden Faktoren für die Entstehung, Durchführung und Verbreitung innovativer Ansätze. Der vorliegende Beitrag konzentriert sich auf die Fragestellung, welche förderlichen Bedingungen und Hemmnisse auf das Wirken und die Verbreitung der Transformationspioniere Einfluss nehmen.

\section{Konzept der Transformationspioniere}

Zur Untersuchung systemischer und gesamtgesellschaftlicher Veränderungsprozesse eröffnet die Transition Theory (Geels 2005; Geels et al. 2007) einen geeigneten Rahmen. Das Modell beschreibt den Einfluss innovativer Nischen auf etablierte Strukturen und Prozesse (Geels et al. 2007). Die Politik-, Orga- nisations- und Raumwissenschaften bieten mit Change Agents oder Promotor/innen (Kristof 2010), Schlüsselakteuren (Kilper et al. 2016) oder Leadership (Nerdinger et al. 2012) akteursorientierte Zugänge für die innovativen Nischen. Diese Ansätze liefern Erkenntnisse über Einfluss und Eigenschaften innovativer Akteure, welche unter dem Begriff „Pioniere des Wandels“ (WBGU 2011) zusammengefasst werden können. Diesen Pionieren wird die Eigenschaft als Initiator/innen oder auch als Treiber/innen gesellschaftlicher Veränderungsprozesse zugesprochen (WBGU 2011). Pioniere können diese Prozesse interaktiv und diskursiv gestalten und begleiten sowie Veränderungen von Lebensstilen oder eine Verschiebung von Werten und kulturellen Normen bewirken (Kilper et al. 2016).

Für den Untersuchungsansatz in dem Forschungsvorhaben Climate Smart City Hamburg / Lokstedt (FKZ 01U R1608B)wurde auf Basis der genannten Diskussionsstränge der Ansatz der Transformationspioniere zugrunde gelegt (Engel et al. 2018). Danach werden Transformationspioniere als Individuen oder kleine Gruppen definiert, die mit ihrem Handeln in Richtung eines gesamtgesellschaftlichen Wandels zur Nachhaltigkeit wirken. Sie haben ihren Lebensstil bereits am Klimaschutz ausgerichtet und entwickeln und realisieren neue Ideen und Ansätze, die auf eine Transformation hinwirken (Engel et al. 2018). Dies bedeutet, dass ihre Projekte und Aktivitäten zur Zielvorstellung einer nachhaltigen Gesellschaft beitragen. Pioniere wirken über einen inkrementellen Wandel hinaus in Richtung einer tief greifenden Transformation, indem sie bestehende Werte, Normen und Handlungsweisen herausfordern. In ihren Aktivitäten wird eine Abkehr von einem „Weiter-wie-bisher" erkennbar (Kristof 2010; WBGU 2011).

\section{Transformationspioniere in Hamburg}

Das Forschungsvorhaben Climate Smart City Hamburg / Lokstedt identifizierte Transformationspioniere aus den drei Handlungsfeldern Energie, Mobilität und Abfall im Stadtteil Lokstedt, im Bezirk Eimsbüttel sowie im Stadtgebiet Hamburg. Als Klimaschutzpioniere können dabei diejenigen Akteure beschrieben werden, die einen Beitrag zu den Zielen des Klimaschutzes in den drei Handlungsfeldern leisten und gleichzeitig über die in Kapitel 2 genannten transformativen Eigenschaften verfügen.

Grundlage des Wirkens vieler der identifizierten Pioniere ist ihre Kritik an der Wegwerfkultur sowie der Energie- und Ressourcenverschwendung, weshalb sie mit ihren Projekten Alternativen zu etablierten Handlungsmustern anbieten. Im Themenfeld Energie beziehen sich die Aktivitäten der Pioniere 
zum Beispiel auf die Thematik energiereduzierter Lebensstile (etwa Fahrraddiscos zur Sensibilisierung des eigenen Umgangs mit der unsichtbaren Ressource Energie), die Finanzierung von Energieeffizienzmaßnahmen in Clubs oder im Einzelhandel, zur Vermietung von Solaranlagen oder zur energetischen Gebäudesanierung in kooperativen Sanierungsgemeinschaften. Mobilitätspioniere bieten zum Beispiel Selbstbauworkshops für Lastenräder an oder verkaufen und vermieten diese, produzieren und vertreiben elektrifizierte Fahrradanhänger und Elektrofahrzeuge (E-Scooter, E-Lieferantenroller, E-Räder) oder bieten einen umweltfreundlichen Logistikservice wie auch umweltfreundliche innerstädtische Last-Mile-Konzepte an. Im Bereich Abfall finden sich Pionieraktivitäten bei Themen wie verpackungslosen Supermärkten, Partys und Netzwerken zum Teilen, Tauschen und Verschenken von Produkten, Vorträgen und Infoabenden zur Lebensstiländerung (etwa „plastikfrei leben“ oder Zero Waste), Repair-Cafés und Co-Working-Spaces oder auch kommerziellen Angeboten von Schmuck, Möbeln und Kleidung aus Reststoffen und Fehlproduktionen.

Die Pioniere sind mit ihren Projekten in unterschiedlichen Organisationsformen aktiv. Diese reichen von innovativen Einzelpersonen über temporäre Gruppierungen und Initiativen, die Keimzellen von Nischeninnovationen sein können, aber eine (noch) relativ geringe Sichtbarkeit haben, bis hin zu einer stärkeren Formalisierung, etwa die Organisation über einen Verein, eine Genossenschaft oder ein Unternehmen. Letztere verändern die Handlungsorientierung und die Handlungsmöglichkeiten der Akteure, da handlungsfähigere Arbeitsformen entstehen und Finanzressourcen Möglichkeitsräume eröffnen, zugleich aber auch arbeitsteilige und teils hierarchische Strukturen als organisatorische Herausforderungen entstehen können. Die Formalisierung kann einen Übergang von der Nische in den Mainstream bedeuten, führt allerdings nicht zwangsläufig zu einer breiteren Diffusion oder erfolgreichen Etablierung des Pioniers beziehungsweise der Pionierinnovation.

\section{Einflussfaktoren auf das Wirken von Transformationspionieren}

Das Wirken von Individuen auf (gesellschaftliche) Veränderungsprozesse wird mit Konzepten wie Change Agent oder Promotor/innen (z. B. Kristof 2010), Schlüsselakteur (z. B. Kilper et al. 2016) oder Leadership (Nerdinger et al. 2012) diskutiert. Die verschiedenen Ansätze haben gemein, dass davon ausgegangen wird, dass Einzelpersonen niemals alleine und unabhängig agieren und wirken, sondern immer eingebettet sind in „soziale Beziehungsgefüge“ (Kilper et al. 2016) und in Abhängigkeit von strukturellen Rahmenbedingungen stehen (Kilper et al. 2016; WBGU 2011).

Auf individueller Ebene werden Transformationspioniere dadurch charakterisiert, dass sie mit ihrem Handeln eine Zielvorstellung einer zukünftigen Gesellschaft verfolgen (Kristof 2010). Sie agieren wert- und zielgeleitet, sind intrinsisch motiviert und haben Freude an Veränderung und dem Meistern von Herausforderungen (Nerdinger et al. 2012; Kristof 2010; Maier et al. 2007). Gleichzeitig verfügen sie über „ausreichend Macht, Ressourcen, Kreativität und Innovations- und Reformbereitschaft" (WBGU 2011) und erkennen und nutzen existierende Gelegenheitsstrukturen (Hoffmann 2013; WBGU 2011). Ihnen wird eine strategische Kompetenz zugesprochen, die es ihnen ermöglicht, sich mit weiteren Pionieren zusammenzuschließen und gezielt Transformationsprozesse $\mathrm{zu}$ initiieren und voranzutreiben (WBGU 2011). Als drei zentrale Eigenschaften beschreibt der WBGU (2011) die „innovative Kapazität“, eine „Gemeinwohlorientierung“ und eine hohe „Fähigkeit, ,Neues‘ zu kommunizieren“ (WBGU 2011). Um Wirkung entfalten zu können, müssen Pioniere den Kontakt und die Zusammenarbeit mit anderen Menschen gestalten (Kristof 2010) und „Identität und das Bewusstsein von Wirkungsmächtigkeit“ (WBGU 2011) schaffen können. Sie benötigen dabei relevantes Fach- und Prozesswissen, um in verschiedenen Handlungsfeldern Veränderungs- und Kooperationsprozesse gestalten zu können (Kristof 2010). Dies ermöglicht es ihnen, vielfältige Lösungsideen für Themenfelder zu entwickeln und abzuschätzen, welche Ideen erfolgsversprechend sind und daher weiterverfolgt werden sollten (Maier et al. 2007). Darüber hinaus spielt auf individueller Ebene insbesondere der Glaube an die eigene Selbstwirksamkeit für die Entwicklung und Umsetzung neuer Ideen eine Rolle (Maier et al. 2007). Diese Selbstwirksamkeit in Kombination mit intrinsischer Motivation, Fach- und Prozesswissen können somit einen Wandel in der Handlungsweise eines Individuums hervorrufen und dieses dazu bewegen, Transformationsprozesse anzustoßen.

Transformationspioniere agieren nicht alleine; sie sind vielmehr in vielfältige soziale Beziehungsgefüge und Netzwerke eingebunden, die sie selbst initiieren beziehungsweise prägen. Innerhalb dieser Gruppen und auch von anderen im selben Bereich werden Pioniere oft als „herausragend“ und „bedeutend“ wahrgenommen (Kilper et al. 2016). In ihren Projekten kann es vor allem die Begeisterungsfähigkeit, ihr fachspezifisches Wissen oder die führende Rolle sein, die sie zu „herausragenden“ Persönlichkeiten und damit zu Transformationspionieren macht (Gailing et al. 2016). Diese Fähigkeiten versetzen sie in die Lage, andere von ihrer Idee überzeugen zu können (Kristof 2010). Durch ihr Handeln schaffen sie es nicht nur, Menschen von ihrer Idee zu begeistern, sondern auch Einfluss auf deren individuelle Lebensstile beziehungsweise kulturelle Werte und Normen zu nehmen, um so im nächsten Schritt Veränderungen zu bewirken (Kilper et al. 2016).

Die Zusammenarbeit in Netzwerken ermöglicht darüber hinaus, individuelle Kompetenzen und Ressourcen verschiedener Personen zu kombinieren, um auch über einen längeren Zeitraum hinweg und gegen Widerstände auf ein Ziel hinarbeiten zu können (Kristof 2010). Das Fach- und Prozesswissen sowie die Beziehungen und Machtfunktionen verschiedener Personen können genutzt werden, um lokale oder regionale Akteursnetzwerke aufzubauen. Innerhalb dieser Netze können in gruppendynamischen Prozessen Herausforderungen 
gemeinsam bearbeitet und voneinander gelernt werden (Bendrien et al. 2000). Durch soziale Lernprozesse und den Austausch zwischen den beteiligten Personen werden Ideen und Inhalte weiterentwickelt. Es entstehen geteilte Normen und es kann zu einer Diffusion nachhaltiger Verhaltensmuster kommen (Bendrien et al. 2000). Die Zusammenarbeit in Gruppen und Netzwerken macht Transformationspioniere wirkungsmächtiger (Kilper et al. 2016).

Institutionelle, politische, ökonomische, historische und geografische Rahmenbedingungen beeinflussen die Aktivitäten von Transformationspionieren positiv wie negativ, können stellenweise aber auch von diesen beeinflusst und (mit-)gestaltet werden (Gailing et al. 2016; Kilper et al. 2016). Innovative Projekte und Ideen stehen immer im Wechselspiel mit exogenen Prozessen und Strukturen (Hoffman 2013).

\section{Förderliche und hemmende Faktoren für Transformationspioniere}

Die nachfolgenden empirischen Befunde basieren auf 20 qualitativen, leitfadengestützten Interviews und sind nach zwei Ebenen gegliedert: Auf der individuellen Ebene der Transformationspioniere finden sich deren persönliche und als förderlich befundene Eigenschaften und Kompetenzen sowie die dazu komplementär zu verstehenden Bedarfe und Barrieren. Auf der Ebene der sozialen Beziehungen werden Aussagen zu Kooperationen, Allianzen und Netzwerken getroffen, die Pionieraktivitäten befördern, aber auch Hemmnisse und Barrieren darstellen können. Bei einigen Aspekten konnten in Bezug auf die Pionieraktivitäten sektorale Unterschiede festgestellt werden, welche im letzten Teilkapitel beschrieben werden.

\subsection{Individuelle Eigenschaften und Kompetenzen}

Die Pioniere wurden nach der Motivation für ihre Vorhaben und dem Entstehungshintergrund, nach ihren als besonders hilfreich empfundenen persönlichen Fähigkeiten sowie Schwierigkeiten in der Projektumsetzung befragt, aber auch nach den Strategien, über die Verbündete und Partner/innen gefunden wurden.

Transformationspioniere verfügen über eine hohe Kommunikationsfähigkeit zur Ansprache und Werbung von Mitstreitenden. Dies zeigt sich darin, dass Pioniere oftmals ein breites persönliches Netzwerk pflegen und Engagierte zu Beginn ihrer Projekte über ihr persönliches Umfeld einbeziehen. In einigen Fällen sind Impulse und Anregungen für Pionierprojekte aus dem persönlichen Netzwerk hervorgegangen. Bei fortgeschrittenen Aktivitäten hat es sich als hilfreich erwiesen, wenn die Pioniere Gruppenprozesse leiten und moderieren können, aber auch über motivierende Eigenschaften und Durchhaltevermögen verfügen, welches sich besonders in Konfliktsituationen und bei Ressourcenknappheit auszahlt. Als Hemmnis zeigt sich, dass die Pioniere teilweise aufwendige Überzeugungsarbeit leisten müssen, wenn sich ihre Projekte und Aktivitäten einer komplexen Thematik widmen und deshalb ei- ner Erklärung bedürfen. Auch nehmen potenziell Engagierte den Problemdruck durch den Klimawandel bisher nicht ausreichend wahr, sodass dieses Argument noch wenig engagementfördernd wirkt.

Die Pioniere zeigen eine besondere Sensibilität für Gelegenheitsfenster und die Fähigkeiten, diese auch zu nutzen. So besteht seit einiger Zeit beispielsweise eine breite öffentliche und mediale Aufmerksamkeit für die Themen Energiewende und Sharing-Economy. Den Pionieren bietet dies eine Basis, ihre Ideen zu platzieren und diese offensiv und kompetent nach außen zu vermitteln. Ein technisches Grundverständnis oder auch handwerkliche Fähigkeiten zeigen sich als förderlich, wenn es darum geht, Mitstreiter/innen oder Sponsor/innen zu motivieren, aber auch bei der Umsetzung der Aktivitäten. Defizite bei persönlichen Kompetenzen beschreiben die Pioniere im Bereich der Rechtsberatung zu den Themen des Steuer-, Arbeits-, Internetrechts sowie des Gründungs- oder Vereinsrechts, die sich zu projektgefährdenden Hindernissen entwickeln können. In der Startphase der Aktivitäten besteht ein Hemmnis darin, für das Innovationsvorhaben die geeignete Rechtsform zu finden.

\subsection{Soziale Beziehungen und Netzwerke}

In diesem Bereich wurden die Pioniere nach ihren Verbündeten gefragt, nach Kompetenzen, die von außen eingeholt werden, der Zusammenarbeit mit weiteren Institutionen und Akteuren, aber auch nach Schwierigkeiten, die durch eine gemeinschaftliche Projektumsetzung entstehen.

Pionierprojekte werden oft von besonders engagierten Einzelpersonen und ehrenamtlich Tätigen getragen, die sich den Projekten oder der Sache sehr verpflichtet fühlen. Bei Bedarf können auch kurzfristig Engagierte aus dem persönlichen Netzwerk heraus gewonnen werden. In einigen Fällen sind die Netzwerke organisch aus den Aktionen heraus entstanden, worüber Referenzen und Vertrauen aufgebaut werden konnten. Allerdings zeigt sich bei verschiedenen Pionieraktivitäten unstetes Engagement und eine mangelnde Bindungsfähigkeit von Personen als Hemmnis. Oft sind Engagierte im Ehrenamt aktiv oder während besonderer Lebensphasen, zum Beispiel während eines freiwilligen ökologischen Jahres oder eines Sabbatjahres. Um ehrenamtliches Engagement in feste Personalstellen zu überführen, fehlen in vielen Fällen die finanziellen Ressourcen. Diese ökonomische Barriere wirkt sich negativ auf die Professionalisierung der Aktivitäten aus und beeinflusst die Entwicklung und ein eventuelles Mainstreaming der Pionierprojekte. Aus fortgeschrittenen Pionierprojekten wurde allerdings teilweise von Missstimmungen unter den Engagierten berichtet, die sich über die Ablösung ehrenamtlicher Aufgaben durch Professionalisierung (z. B. Mini- oder Medi-Jobs) ergeben können. Gefahren liegen darin, die Ehrenamtlichen zu demotivieren, wenn andere Personen für vergleichbare oder benachbarte Aufgaben ein Gehalt beziehen, sich Veränderungen in der Intensität des Engagements oder auch in projekthemmenden Gruppenprozessen ergeben (z. B. Spaltung von 
Beschäftigung und Ehrenamt). Die finanziellen Ressourcen für diese Aufgabenüberführung werden überwiegend aus öffentlichen Fördermitteln getragen. Schwierigkeiten zeigten sich teils bei der Fortführung von Aufgaben nach Auslaufen dieser Fördermittel. In dieser Phase sind individuelle Kompetenzen der Pioniere hilfreich, etwa eine hohe Sensibilität für Gruppenprozesse und die Fähigkeit, motivierend auf das Team zu wirken.

Förderlich für die Pionieraktivitäten ist der Anschluss an ein übergreifendes, meist bundesweites Netzwerk. Hierüber können beispielweise ideelle Unterstützung oder erste Kundschaft angesprochen werden, die mit ihrem Namen Vertrauen schaffen, sodass darüber weitere Mitglieder, Engagierte, Sponsor/innen oder Kunden/innen geworben werden können. Während die Werbung für die Pionieraktionen in der Regel über das persönliche Netzwerk, Flyer, Plakate und Events erfolgt, konnten vor allem die Abfall- und Mobilitätspioniere über Netzwerke Wettbewerbe und Stipendien erschließen. Diese brachten mediale Aufmerksamkeit und Marketing für die Pioniervorhaben. In wenigen Fällen gab es eine Kompetenzvermittlung (Coaching) oder auch Geldpreise. Insgesamt berichten die Pioniere, dass Öffentlichkeitsarbeit und Marketing die Bereiche sind, die aus finanziellen wie personellen Gründen immer wieder als Erstes in den Hintergrund rücken.

Alle Pioniere haben Engpässe bei passenden Räumlichkeiten zum Austausch und zur Durchführung ihrer Aktivitäten als Hemmnis erlebt. Nur in wenigen Fällen konnten entsprechende Räumlichkeiten über Kooperationspartner/innen oder das eigene Netzwerk erschlossen werden.

\subsection{Sektorale Unterschiede}

Bei einigen Aspekten unterschieden sich die Einschätzungen der Pioniere je nach Themenfeld, sodass sich sektorspezifische Befunde beschreiben lassen. Bei den Mobilitäts- und Abfallpionieren zeigen sich beispielsweise Kooperationen mit verwandten Projekten und mit Wirtschaftsunternehmen als besonders förderlich. Hierüber können günstig oder auch kostenfrei Materialressourcen erschlossen und auch Unterstützung durch spezifische Fachexpertise eingeholt werden. Bei den Energie- und Abfallpionieren ist der Kontakt zu Fachleuten, die bei technischen Fragen zur Verfügung stehen, hilfreich für den Erfolg und das Vorankommen der Projekte.

Das Thema Konkurrenz wird bei den Mobilitäts- und Abfallpionieren teilweise positiv angesprochen beziehungsweise nicht als negativer Wettbewerb verstanden. Mehr als die Hälfte der interviewten Pioniere betonen, dass sie es als motivierend und positiv empfinden würden, wenn in anderen Stadtteilen ihre Projektideen oder Aktionen gleich oder ähnlich durchgeführt würden. Die Pioniere unterstreichen die positive Kraft einer gesellschaftlichen Bewegung, die sich über die Verbreitung ihrer Aktionen äußert. Bei den Energiepionieren wird das Thema Konkurrenz differenziert gesehen, da bei der Umsetzung der Energiewende die Flächenverfügbarkeit eine wesentliche Rolle spielt und diese Ressource in der Stadt Hamburg knapp ist. Die Konkurrenz um Flächen spielt jedoch vorran- gig bei den eher etablierten Akteuren im Pfad der Energieerzeugung eine Rolle und weniger in den drei anderen Pfaden der Energiewende (Energiesuffizienz, Energieeffizienz, Sektorkopplung) oder bei den Akteuren, die weniger formalisiert sind.

Der Kontakt zu Macht- und Fachpromotor/innen stellt sich in der Wichtigkeit unterschiedlich dar. Während bei den Energie- und Mobilitätspionieren der Kontakt zu Machtpromotor/ innen für das Gelingen einiger Projekte wesentlich war, spielen diese bei den Abfallpionieren eine geringere Rolle. Dagegen erwiesen sich hier die Kontakte zu Fachpromotor/innen mit speziellem Know-how als besonders förderlich. Hintergrund könnte sein, dass die Energie- und Mobilitätswende mit entsprechenden formalen Regelungen differenzierter ausgestaltet und bereits weiter fortgeschritten ist. Aus diesem Grund kommt den Machtpromotor/innen beim Vorankommen der Energie- und Mobilitätspioniere eine wichtigere Rolle zu als den Fachpromotor/innen.

\section{Beiträge der Pioniere zu einer klimafreundlichen Stadtgesellschaft}

Die in Hamburg identifizierten Transformationspioniere zeigen verschiedenste innovative Lösungsansätze für den Klimaschutz in Städten. Einige der Pioniere fordern etablierte Werte und Normen heraus, indem sie zum Beispiel über suffizienzorientierte Lebensweisen oder zum bewussten Umgang mit Ressourcen aufklären. Mit ihren experimentellen Projekten und knappen Ressourcen gehen die Pioniere oftmals ein hohes Risiko des Scheiterns ein. Ihr Erfolg hängt von ihren persönlichen Eigenschaften, ihren individuellen Kompetenzen und dem besonderen Engagement ab, aber auch davon, die richtigen Verbündeten und Partner/innen (Fach- und Machtpromotor/innen) zu finden. Für eine Transformation zu einer klimafreundlichen Stadtgesellschaft geben die Pioniere wertvolle Impulse, benötigen aber eine besondere Art der Innovationsförderung, um in ihrem Wirken unterstützt zu werden. Die Transformation könnte befördert werden, indem zum Beispiel die Risiken der Pioniere reduziert und durchlässigere Strukturen geschaffen werden, die es ermöglichen, den Status quo in den jeweiligen Handlungsbereichen des Klimaschutzes zu verändern. Dazu könnte beispielsweise ein gezielter Austausch zwischen Pionieren und Institutionen beitragen, um innovative Ideen zu platzieren und zu testen, aber auch um die Zugänglichkeit zu erforderlichen Ressourcen und Schlüsselpersonen zu erleichtern.

Die empirischen Ergebnisse haben einen hohen Bedarf an niedrigschwelligen Beratungsangeboten angedeutet. Hierfür könnten Angebote einer (möglichst kostenfreien) Beratung für Organisationsfragen (Vereinsrecht, Betriebsrecht) oder für Versicherungsfragen bereitgestellt werden. Auch könnten Beratung und Workshops für die Beantragung von öffentlichen Fördermitteln helfen. Hilfreich wäre es für die Pionierprojekte, wenn zum Beispiel die Stadt flexible Räumlichkeiten (auch mit Werkstattoption) zu günstigen Konditionen für die Startphase 
der Pionierprojekte bereitstellen würde. Gerade Orte, an denen ungezwungen Zusammenarbeit möglich ist, um eine Gruppe zu bilden, gemeinsam Ideen reifen zu lassen und diese auch ausprobieren zu können, wird von den Pionieren als Bedarf beschrieben.

Die Fallstudie zu den Transformationspionieren weist auch auf das Defizit hin, dass die öffentlichen Strukturen zur Innovationsförderung vorrangig auf Finanzierungshilfen ausgerichtet sind, welche die Transformationspioniere kaum erreichen, da spezifische Voraussetzungen und Bedingungen gefordert werden. Eine kommunale Förderung, die auf die spezifischen Bedarfe der Transformationspioniere ausgerichtet ist, könnte sowohl die Startphase als auch die Etablierung der Pionieraktivitäten erleichtern. Der Mehrwert der Projekte für den Klimaschutz und für das Gemeinwohl könnte als Kriterium herangezogen werden.

Die finanzielle Unterstützung stellt allerdings nur einen Aspekt möglicher förderlicher Rahmenbedingungen für Transformationspioniere dar. Weiterer Forschungsbedarf bezieht sich auf Fragen der sozialen, institutionellen und räumlichen Einbettung und Verflechtung der Pionierprojekte. Eine Analyse der Entwicklung der Pionierinnovationen könnte - unter Berücksichtigung der Erkenntnisse der klassischen Innovationsforschung - Aufschluss darüber geben, wie der Übergang vom Nischenprojekt in den gesellschaftlichen beziehungsweise ökonomischen Mainstream gelingt und welche Strategien und Anforderungen damit verbunden sind. Darüber hinaus sollte die Wirkung der Pionierprojekte vertiefend untersucht werden, um herauszuarbeiten, inwiefern und wie diese zu einem Wandel von kulturellen Normen und Werten in Richtung einer klimafreundlichen und nachhaltigen Stadtgesellschaft beitragen können.

\section{Literatur}

Bendrien, J./Engellhandt, C./Prose, F. (2000): Kommunale Akteure und soziale Netze - Ein sozialpsychologisches Rahmenmodell zur Analyse kommunalen Klimaschutzes. In: Böde, U./Gruber, E. (Hrsg.): Klimaschutz als sozialer Prozess. Berlin, Springer VS. 13-61.

Berkhout, F./Raven, R./Schot, J. (2012): Space and scale in socio-technical transitions. In: Environmental Innovation and Societal Transitions 4: 63-78.

Bulkeley, H./Castán Broto, V./Maassen A. (2011): Governing urban low carbon transitions. In: Bulkeley, H./Castán Broto, V./Hodson, M./ Marvin S. (Hrsg.): Cities and Low Carbon Transitions. New York, Routledge. 29-53.

Engel, T./Klindworth, K./Knieling, J. (2017): Green Growth oder Postwachstum? In: RaumPlanung 191: 23-28.

Engel, T./Klindworth, K./Knieling, J. (2018): Einflüsse von Pionieren auf gesellschaftliche Transformationsprozesse im Handlungsfeld Energie. In: Franz, H.-W./Kaletka, C. (Hrsg.): Soziale Innovationen lokal gestalten. Berlin, Springer VS. 215-231.

Fenzl, T./Mayring, P. (2014): Qualitative Inhaltsanalyse. In: Baur, N./Blasius, J. (Hrsg.): Handbuch Methoden der empirischen Sozialforschung. Berlin, Springer VS. 543-556.

Gailing, L./lbert, O. (2016): Schlüsselfiguren: Raum als Gegenstand und Ressource des Wandels. In: Raumforschung und Raumordnung 74/5: 391-403.
Geels, F. W. (2011): The role of cities in technological transitions. Analytical clarifications and historical examples. In: Bulkeley, V./Castán Broto, V./ Hodson, M./Marvin, S. (Hrsg.): Cities and Low Carbon Transitions. New York, Routledge. 13-28.

Geels, F. W./Schot, J. (2007): Typology of sociotechnical transition pathways. In: Research Policy 36/3: 399-417.

Geels, F. W. (2005): Processes and patterns in transitions and system innovations. Refining the co-evolutionary multi-level perspective. In: Technological Forecasting \& Social Change 72/6: 681-696.

Gibbs, D./O'Neill, K. (2014): The green economy, sustainability transitions and transition regions: a case study of Boston. In: Geografiska Annaler Series B. Human Geography 96/3: 201-216.

Hoffman, J. (2013): Theorizing power in transition studies: the role of creativity and novel practices in structural change. In: Policy Sciences 46/3: 257-275.

Kilper, H./Christmann, G. B. (2016): Schlüsselfiguren als Triebkräfte in der Raumentwicklung - konzeptionelle Überlegungen für ein analytisches Modell. In: IRS AKTUELL 87: 5-7.

Kristof, K. (2010): Models of Change. Einführung und Verbreitung sozialer Innovationen und gesellschaftlicher Veränderungen in transdisziplinärer Perspektive. Zürich, vdf Hochschulverlag.

Maier, G. W./ Streicher, B./ Jonas, E./ Frey, D. (2007): Innovation und Kreativität. In: D. Frey / von Rosenstiel, L. (Hrsg.): Enzyklopädie der Psychologie. Themenbereich D, Praxisgebiete: Serie 3, Wirtschafts-, Organisations- und Arbeitspsychologie: Vol. 6. Wirtschaftspsychologie. Verlag Hogrefe Göttingen. 809-855.

Nerdinger, F. W./Pundt, A. (2012): Transformationale Führung - Führung für den Wandel? In: Grote, S. (Hrsg.): Die Zukunft der Führung. Berlin, Springer. 27-45.

O'Brien, K. (2012): Global environmental change II. From adaptation to deliberate transformation. In: Progress in Human Geography 36/5: 667-676.

Seyfang, G./Haxeltine, A. (2012): Growing grassroots innovations: exploring the role of community-based initiatives in governing sustainable energy transitions. In: Environment and Planning C. Government and Policy 30/3: 381-400.

Smith, A. (2011): Community-led urban transitions and resilience: performing Transition Towns in a city. In: Bulkeley, V./Castán Broto, V./Hodson, M./ Marvin, S. (Hrsg.): Cities and Low Carbon Transitions. New York, Routledge. 159-177.

WBGU (Wissenschaftlicher Beirat der Bundesregierung Globale Umweltveränderungen) (2011): Welt im Wandel. Gesellschaftsvertrag für eine Große Transformation. Hauptgutachten. Berlin, WBGU.

WBGU (Wissenschaftlicher Beirat der Bundesregierung Globale Umweltveränderungen) (2016): Der Umzug der Menschheit: Die transformative Kraft der Städte. Berlin, WBGU.

Welzer, H./Sommer, B. (2014): Transformationsdesign. Wege in eine zukunftsfähige Moderne. Transformationen. München, Oekom.

\section{AUTOR/INNEN + KONTAKT}

Toya Engel ist wissenschaftliche Mitarbeiterin und Dr.Jörg Knieling ist Professor im Fachgebiet Stadtplanung und Regionalentwicklung an der HafenCity Universität Hamburg.

HafenCity Universität Hamburg, Überseeallee 16 , 20457 Hamburg. Tel.: +49 40428274610 , E-Mail: toya.engel@hcu-hamburg.de, joerg.knieling@hcu-hamburg.de

Katharina Klindworth ist Mitarbeiterin bei "Our Common Future Consulting“.

Our Common Future Consulting, Osterstraße 124, 20255 Hamburg. Tel.: +494046642440 , E-Mail: katharina.klindworth@ocfc.de, Website: www.ocfc.de

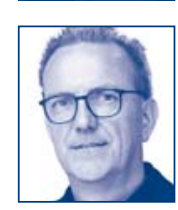

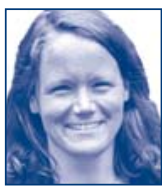

